\title{
CONTINUOUS MEAN DEMAND FUNCTIONS DERIVED FROM NON-CONVEX PREFERENCES*
}

\author{
Egber: DIERKER, Hildegard DIERKER and Walter TROCKEL \\ Unicersity of Bonn, Sonderforschungsbereich. 21, 5300 Bonn 1. Germany.
}

Received September 1980

In this paper we show that for a largo subset of utility functions in the space of all $C^{*}$ urility functions and for all prices the mean demand of those consumers whose taste is represented by a given utility function in that subset is uniquely determined. This implies that for a large set of economies mean demand is a continuous function. Our analysis uses derivatives of first and of higher order. The result is essentially a consequence of the multijet transversality theorem.

\section{Introduction}

The price mechanism is commonly believed to achieve the consistency of individual decisions in a purely competitive economy. However, the complete coherence of individual decisions based on an equilibrium price system is unlikely to be obtained if tite aggregate decision is not well-determined by the price system. In the presence of preference non-convexities, individual decisions are not necessarily determined by the price system and it is questionable whether the knowledge of equilibrium prices is sufficient to obtain compatibility of individual decisions.

For the concept of an equilibrium price system to obtain its full power, one would like to have a continuously differentiable mean demand furctinn. Our approach in this paper has indeed been motivated by the ufiestion of when mean deniand can be expected to be differentiable. In another paper [Dierker et al. (1980)] we show that for a fixed preference relation, aggregation with respect to a continuous income distribution leads to a continuously differentiable demand function except for prices in a closed nall set. But in this aull set of prices demand need not be unique.

In this paper we show that the mean demand of ail consumers is a uniquely determined bundle for all price systems if tastes are represented by utility functions belonging to a large subset of a given class. This together

*This paper was written while E. Dierker was on sabbatical leave at the University of California, Berkeley. The authors have benefited from conversations with several colleagues at Bonn and Berkeley. 
with the well-known upper hemi-continuity of the mean demand correspondence implies that the mean demand of the consumption sector is a continuous function. Our analysis relies heavily on the use of cerivatives of first and of higher order. The result obtained is essentially a consequence of the multijet transversality theorem [see Golubitsky and Guillemin (1973, p. 57)]. ${ }^{1}$ For the study of continuity of mean demand without the use of derivatives, see Mas-Colell and Neuefeind (1977, sect. 5).

The continuity of mean demand has been studied in the framework of differentiable utility functions by Sondermann $(1975,1976,1980)$, and by Araujo and Mas-Colell (1978). A major difference between their work and ours is that they stipulate a manifold structure on the space of preferences considered, a stipulation which we want to avoid. The manifold structure is used to express the notion of dispersed preferences. Dispersion of preferences is not needed in the present paper because the uniqueness of mean demand is essentially obtained by integration with respect to wealth keeping preferences fixed. Variation of preferences becomes important, though, if the differentiability of mean demand is studied.

\section{Model and result}

There are $l \geqq 2$ commodities. The commodity space is $R^{l}$. The consumption set of every consumer is

$$
X=\left\{x \in R^{l} \mid x \gg 0\right\}
$$

We consider prices in

$$
S=\left\{p \in R^{l} \mid p \gg 0,\|p\|=1\right\} .
$$

The norm is assumed to be $C^{x}$ on the positive orthant of $R^{l}$. To be specifis, we choose the Euclidean norm.

Let $\mathscr{P}$ be the space of preference orderings which are representable by $C^{x}$ utility functions $u: X \rightarrow R$ satisfying assumptions (U.1), (U.2), and (U.3) below:

(U.1) $D u(x) \gg 0$ for all $x \in X$ (monotonicity).

(U.2) The closure of each indifference hypersurface is contained in $X$ (a boundary assumption made to keep demand inside $X$ ).

Let $g(x)=D u(x)\|D u(x)\|^{-1}$. We postulate:

(U.3) $g: X \rightarrow S$ has everywhere maximal rank, i.e., rank $l-1$.

\footnotetext{
'We would like to mention that the multije: transversality theorem has been applied by Sondermann (1980) to derive results which are closely related to ours.
} 
This assumption allows indifference hypersurfaces to have Gaussian curvature zero. However, it rules out the case of two or more principle curvatures vanishing simultaneously.

Let $\mathscr{U}$ be the space of $C^{*}$ utility functions $u: X \rightarrow R$ satisfying (U.1), (U.2) and (U.3), endowed with the $C^{*}$ Whitney topology.

A neighborhood basis for $u \in \mathscr{U}$ in the $C^{k}$ Whitney topology is obtained by taking, for any continuous mapping $\delta: X \rightarrow] 0, \propto[$, the set of those utility functions for which, at every $x \in X$, the $C^{k}$ distance $o u$ is smaller than $\delta(x)$. The $C^{x}$ Whitney topology is used for simplicity's sake.

Let $w \in] 0, x$ [ denote the wealth of a consumer. The demand of an agent with wealth $w \in] 0, x[$ and preference ordering $\lesssim \in \mathscr{p}$ at price system $p \in S$ is

$$
\varphi(\lesssim, p, w)=\{x \in X \mid p \cdot x \leqq w, y>x \curvearrowright p \cdot y>n\} .
$$

For given $\lesssim \epsilon . \mathcal{P}$, let the wealth distribution be described by a probability measure $\delta_{-}$on $\mathscr{B}(] 0, \infty[), \not B$ denoting the Borel $\sigma$-algebra, such that the mean wealth is finite, i.e., $\int w \delta_{s}(\mathrm{~d} w)<x$. We assume that $\delta_{5}$ is absolutely continuous with respect to the Lebesgue measure $i$. Let $h$, denote the density of $\delta_{\leqslant}$with respect to $\lambda$. Then, for the given preference crdering $\leqslant \in$. P. the mean demand at $p \in S$ with respect to wealth is

$$
\Phi(\lesssim, p)=\int_{0}^{x} \varphi(\lesssim, p, w) h_{\leqslant}(w) \mathrm{d} i
$$

The integral of a correspondence $\psi$ from the measure space $(\Omega, \sigma, v)$ into $\boldsymbol{R}^{m}$ is defined as follows. Denote by $\mathscr{H}_{\psi}$, the set of rintegrable functions $s: \Omega$ $\rightarrow \boldsymbol{R}^{m}$ such that $s(w) \in \psi(w)$ i $\cdots$ a.e. in $\Omega$. Then the set

$$
\left\{j: i v \in \boldsymbol{R}^{m} \mid s \in \mathscr{L}_{\psi}\right\}
$$

is called the integral of $\psi$ [cf. Hildenbrand (1974)].

Theorem. The space "I has a residual subset "I res such that each element in $\|_{\text {res }}$ represents a preference ordering $\lesssim$ for which the mean demand $\Phi(\lesssim . \cdot): S$ $\rightarrow X$, given $\lesssim$, is a continuous function.

Remark. Let $\mu$ be a measure on $\mathscr{P}$ such that $\mu$-almost all preferences are representable by utility functions in $\|_{\text {re }}$. Then the mean demand $F: S \rightarrow X$ given by

$$
F(p)=\int \Phi(\lesssim, p) \mu(\mathrm{d} \lesssim)
$$

is a continuous function. 


\section{Proofs}

Let $u \in \mathscr{U}$ be given. Let $\kappa(x)$ denote the Gaussian curvature of the indifference hypersurface $u^{-1}(u(x))$ at $x \in X$.

Lemma. Let $u \in \mathbb{i l}$ and $p \in S$ be given. Then

$$
\{w=g(x) \cdot x \mid g(x)=p, \kappa(x)=0\} \quad \text { is null. }
$$

Proof. The proof exploits the assumption that $g$ has maximal rank everywhere on $X$. Let $p \in \operatorname{im}(g)$. Then $g^{-1}(p)$ is a cne-dimensional differentiable manifold. Assume $\kappa(x)=\mathrm{J}$ for $x \in g^{-1}(p)$. The tangent space $T_{x} g^{-1}(p)$ is given by the kernel of $D g(x)$. Since $\kappa(x)=0$, the kernel of $D g(x)$ is contained in $T_{x}\left(u^{-1}(u(x))\right)$. It follows that $g^{-1}(p)$ is tangent in $x$ to the indifference hypersurface $u^{-1}(u(x))$ and therefore to the budget hyperplane through $x$ with normal vector $p$. Therefore, a point $x \in g^{-1}(p)$ with $\kappa(x)=0$ is a critical point of the mapping $g^{-1}(p) \rightarrow R, x \mapsto g(x) \cdot x$. The set of critical values of this mapping is a null set. Q.E.D.

Remark. The lemma permits one to neglect points with vanishing Gaussian curvature when demand is integrated with respect to wealth for a given preference ordering. Assumption (U.3) allows us to achieve this result in a simple way. Points with vanishing Gaussian curvature can be considered as points where a catastrophe occurs. One might hope that the use of catastrophe theory allows a considerable weakening of (U.3).

We want to show that there exists a residual subset $\mathscr{U}_{\text {res }}$ of $\mathscr{U}$ such that, for any $u \in \mathscr{U}_{\text {re }}$ and for any $p \in S$, demand is single-valued except for a set of isolated points $w \in] 0, \infty$ [ and except for the null set of $w$ 's described in the lemma. To show this we use the notion of a jet and a powerful transversality theorem. See Golubitsky and Guillemin (1973, ch. 2, §2, §4) for the terminology and statements to be used.

is: fore we get involved with the technicalities, we give an intuitive sketch of the basic idea. Suppose $u \in \mathscr{U}$ is given and the demand at $(p, w)$ does not contain a point whose associated indifference surface has vanishing Gaussian curvature. The demand at $(p, w)$ is contained in the intersection of $g^{-1}(p)$ and the budget hyperplane $B_{p, w}$ corresponding to $(p, w)$. The intersection is transversal because of the non-vanishing Gaussian curvature. Therefore $B_{p, w} \cap g^{-1}(p)$ consists of isolated puints. Indeed, $\#\left(B_{p, w} \cap g^{-1}(p)\right)<\infty$ because of the boundary assumption (U.2). Fach point in $B_{p, w} \cap g^{-1}(p)$ can be traced locally if $w$ is varied $p$ remaining fixed. That is to say, there are $\varepsilon$ $>0$ and smooth functions $\left.h_{1}, \ldots, h_{r}:\right] w-\varepsilon, w+\varepsilon\left[\rightarrow X, r=\#\left(B_{p, w} \cap g^{-1}(p)\right)\right.$ such that, for $\left.w^{\prime} \in\right] w-\varepsilon, w+\varepsilon\left[\right.$, we have $B_{p, w^{\prime}} \cap g^{-1}(p)=\left\{h_{1}\left(w^{\prime}\right), \ldots, h_{r}\left(w^{\prime}\right)\right\}$. 
Suppose $x_{1}=h_{1}(w)$ and $x_{2}=h_{2}(w)$ are demanded at $(p, w)$. Then, in particular, $u\left(x_{1}\right)=u\left(x_{2}\right)$ and $D u\left(x_{1}\right)$ is proportional to $D u\left(x_{2}\right)$. If $D u\left(x_{1}\right)$ exceeds $D u\left(x_{2}\right)$, then a slight increase of wealth from $w$ to $w^{\prime}$ prevents $h_{2}\left(w^{\prime}\right)$ from belonging to the demand at $\left(p, w^{\prime}\right)$, because $u\left(h_{1}\left(w^{\prime}\right)\right)>u\left(h_{2}\left(w^{\prime}\right)\right)$. Similarly, a slight decrease of wealth from $w$ to $w^{\prime \prime}$ prevents $h_{1}\left(w^{\prime \prime}\right)$ from belonging to the demand at $\left(p, w^{\prime \prime}\right)$.

However, the case $D u\left(x_{1}\right)=D u\left(x_{2}\right)$ cannot be excluded, not even in the case of only two commoaities. Therefore, assume now $D u\left(x_{1}\right)=D u\left(x_{2}\right)$. Then one is led to consider the second order variation of $u$ at $x_{i}$ along $h_{i}$. If the second order increase of $u$ at $x_{1}$ along $h_{1}$ exceeds that of $u$ at $x_{2}$ along $h_{2}$, then a similar reasoning as above shows that a slight variation of wealth prevents one of the commodity bundles from belonging to the demand set. If the first and the second order increase of utility at $x_{1}$ along $h_{1}$ and of $x_{2}$ along $h_{2}$ happen to coincide, apply a similar argument to the third crder increase of utility, etc. The condition that all utility increares up to the order of $k$ coincide becomes more and more restrictive when $k$ grows. It turns out that not all utility increases up to the order of $l$ can coincide for a residual set of unility functions. However, one can let the exceptional set of utility functions shrink further by taking derivatives of all orders into account.

To make the preceding reasoning more precise, let $J^{l+1}(X, R)$ be the space of $(l+1)$-jets from $X$ to $R, l \geqq 2$. This space can be conceived of as an open subset of a Euclidean space by describing an $(l+1)$-jet with source in the open set $X \subset R^{l}$ by the coefficients of the associated Taylor polynomial of order $l+1$. Let $x$ be the source of $\sigma \in J^{l+1}(X, R)$. Let $\sigma$ be represented by $u: X \rightarrow R$. We have to look at non-degener:te local utility maximizers subject to the budget constraint. The point $x \in \mathcal{L}$ is a non-degenerate local utility maximizer of $u$ subject to the budget constraint iff $\left.D^{2} u(x)\right|_{\text {ker } D k(x)}$ is negative definite. Non-degeneracy of the local maximizer $x$ does only depend on the 2-jet of $u$ at $x$. Let $O \subset J^{l+1}(X, R)$ consist of those $(l+1)$-jets $\sigma$ which are represented by utility functions having a non-degenerate local maximum subject to the budget constraint at the source of $\sigma$. Note that $\mathbb{C}$ is open.

As suggested by the intuitive sketch of the argument above, we are going to consider indirect utility. Therefore we map $\mathcal{O}$ into $J^{l}(S \times] 0, \infty[, R)$. This space can also be conceived of as an open subset of a Euclidean space by identifying $p=\left(p_{1}, \ldots, p_{l}\right) \in S$ with $\left(p_{1}, \ldots, p_{l-1}\right) \in R^{l-1}$. Let $\sigma \in \mathcal{O}$ be represented by a function $u$. Then there is a neighborhood $U$ of the source $x$ of $\sigma$ such that the function $d$, defined by $d\left(x^{\prime}\right)=\left(g\left(x^{\prime}\right), g\left(x^{\prime}\right) \cdot x^{\prime}\right)$, maps $U C^{x}$. diffeomorphically onto a neighborhood $V$ of $(p, w)=(g(x), g(x) \cdot x)$ in $S \times] 0, \infty\left[\right.$. Assign the $l$-jet represented by $u \circ d^{-1}$ at $(p, w)$ to $\sigma \in \mathcal{C}$. This defines a mapping $f: 0 \rightarrow J^{l}(S \times] 0, \infty[, R)$, provided that the $l$-jet represented by $u \circ d^{-1}$ at $(p, w)$ is independent of the representation of $\sigma$ by $u$.

To check this independence, let $\tilde{u}$ be another representative of $\sigma$. That is to say $\tilde{u} \sim_{\imath+1} u$ at $x$, the symbol $\sim_{k}$ denoting contact of order $k$. It follows that 
$d \sim d$ at $x$, where $d\left(x^{\prime}\right)=\left(D \tilde{u}\left(x^{\prime}\right)\left\|D \tilde{u}\left(x^{\prime}\right)\right\|^{-1}, \quad D \tilde{u}\left(x^{\prime}\right)\left\|D \tilde{u}\left(x^{\prime}\right)\right\| \cdot x^{\prime}\right)$. Hence $d^{-} \circ d \sim, d^{-1} \circ d=i d$ at $x$. Therefore $d^{-1}=d^{-1} \circ d \circ d^{-1} \sim, d^{-1}$ at $(p, w)$. This implies $u \circ d^{-1} \sim, u \circ d^{-1}$ at $(p, w)$.

Next we want to check that $f$ is differentiable. The l-jet represented by $d$ at $x$ depends differentiably on $\sigma$. The partial derivatives of $d^{-1}$ of order $\leqq k$ can be written as rational functions of the partial derivatives of $d$ of order $\leqq k$. Hence the $l$-jet represented by $d^{-1}$ at $(g(x), g(x) \cdot x)$ depends differentiably on $\sigma$. It follows that the $l$-jet represented by $u \circ d^{-1}$ at $(g(x), g(x) \cdot x)$ depends differentiably on $\sigma$.

We are interested in the way indirect utility varies with respect to wealth at a fixed price system. Therefore let $\pi$ map $J^{l}(S \times] 0, \infty[, R) \rightarrow R^{2 l+1}$ as follows. Assuciate with $\left(p, w, v(p, w), D v(p, w), \ldots, D^{l} v(p, w)\right)$, which represents an element of $J^{l}(S \times] 0, \infty[, R)$, the vector $\left(p_{1}, \ldots, p_{l-1}, w, v(p, w)\right.$, $\left.D_{w} l(p, w) \ldots, D_{w}^{l} v(p, w)\right) \in R^{2 l+1}$, where $D_{w}^{k}$ denotes the $k$ th partial derivative with respect to $w$.

We want to show that $\pi \circ f: C \rightarrow R^{2 l+1}$ is a submersion. Write the tangent space at $\pi(f(c))$ as $R^{l} \times R^{l+1}$. First we show by induction that $\{0\} \times R^{l+1}$ is contained in $\operatorname{im} T_{\sigma}(\pi \circ f)$. For that purpose we rescale the utility function $u$ representing $\sigma$ in a neighborhood of $x=$ source of $\sigma$. Consider $\hat{u}\left(x^{\prime}\right)=u\left(x^{\prime}\right)$ $+\left(u\left(x^{\prime}\right)-u(x)\right)^{\prime}$, where $x^{\prime}$ is close to $x$. Put $v=u \circ d^{-1}$ and $\hat{v}=\hat{u} \circ d^{-1}$ and let (p. $w)=(g(x), g(x) \cdot x), \quad\left(p^{\prime}, w^{\prime}\right)=\left(g\left(x^{\prime}\right), g\left(x^{\prime}\right) \cdot x^{\prime}\right)$. Then $\hat{v}\left(p^{\prime}, w^{\prime}\right)-v\left(p^{\prime}, w^{\prime}\right)$ $=\left(v\left(p^{\prime}, w^{\prime}\right)-v(p, v)\right)^{l}$. Iterated partial differentiation of $\hat{v}-v$ at $(p, w)$ with respect to $w$ yieids that $\left(0, \ldots, 0, x_{1}\right) \in R^{2 l+1}$ is contained in $\operatorname{im} T_{\sigma}(\pi \circ f)$ for $x_{1}=0$. Similarly, adding $\left(u\left(x^{\prime}\right)-u(x)\right)^{l-1}$ to $u\left(x^{\prime}\right)$ yields that $\left(0 . \ldots, 0, x_{2}, x\right) \in \operatorname{im} T_{\sigma}(\pi \circ f), \alpha_{2} \neq 0$. Repeating this argument $l+1$ times, always lowering $k$ in $\left(u\left(x^{\prime}\right)-u(x)\right)^{k}$ by one additional unit until $k=0$, one obtains that $: 0 ; \times R^{l+1}=\operatorname{im} T_{\sigma}(\pi \circ f)$. Next, a sriall translation of the commodity spact in a direction not perpendicular to $p$ shows that $\{0\} \times R \times R^{l+1}$ $=\operatorname{im} T_{\sigma}(\pi \circ f)$. Finally, rotate the commodity space around $x$ to see that $R^{21+1} \subset \operatorname{im} T_{\sigma}(\pi \circ f)$.

Let $J_{2}^{l+1}(X, R)$ denote the 2 -fold $(l+1)$-jet bundle and put

$$
\mathcal{C}_{2}=(\mathcal{C} \times \mathcal{C}) \cap J_{2}^{l+1}(X, R)
$$

$G_{2}$ is an open subset of $J_{2}^{l+1}(X, R)$. Define a mapping $\mathbb{C}_{2} \rightarrow R^{2 l+1}$ by assigning $\pi(f(\sigma))-\pi(f(\tau))$ to $(\sigma, \tau) \in \mathbb{C}_{2}$. The origin in $R^{2 l+i}$ is a regular value of this mapping, since $\pi \circ f$ is a submersion. The inverse image of the origin is a submanifuld $W$ of $c_{2}$ and hence of $J_{2}^{l+1}(X, R)$ of codim $2 l+1$. According to the multijet transversality theorem [cf. Golubitsky-Guillemin (1973, p. 57)] there is a residual subset of $C^{x}(X, R)$ such that, for $u$ in this subset, $j_{2}^{l+1} u$ intersects $W$ transversally. Since $\operatorname{dim}(X \times X)=2 l<\operatorname{codim} W$, transversal intersection amounts to empty intersection. Observe that $\mathbb{Z}$ is 
open in $C^{*}(X, R)$. Therefore. for $u$ in a residual subset $\|_{r e}$. of $\|$, we have $j_{2}^{l+1} u \cap W=\emptyset$.

It remains to show that, for any utility function $u$ with $j_{2}^{l+1} u \cap W=()$ and for any $p \in S$, the mean demand aggregated with respect to weaiti is singlevalued. According to the lemma it is sufficient to show that the set $N$ $=\{w \in] 0, x[\mid u$ has no degenerate, but several non-degenerate maximizers on $B_{p . w}$ ' consists of isolated points. Assume the contrary. Let $\left(w_{n}\right)$ be a sequence in $N$ converging to $w \in N$. Let $x_{n}^{1}$ and $x_{n}^{2}$ be two different utility maximizers on $. B_{p}, w_{n}$. Without restriction we assume that $\left(x_{n}^{1}\right)$ and $\left(x_{n}^{2}\right)$ converge to $x^{1}$ and $x^{2}$. The upper hemi-continuity of the demand correspondence yields that $x^{1}$ and $x^{2}$ are utility maximizers on $B_{p . w}$. Since $w \in N$, we have $k\left(x^{i}\right) \neq 0$ and therefore $x^{1} \neq x^{2}$. Denote the indirect utilit: functions defined locally at $x^{1}$ resp. $x^{2}$ by $l^{1}$ resp. $l^{2}$. As $i_{2}^{l+1} u \cap W=0$, there exists $\bar{k}, 1 \leqq \bar{k} \leqq l$, such that $D_{w}^{k} \cdot l^{1}(p, w)=D_{1}^{k} \cdot 2(p, w)$ for $1 \leqq k<\bar{k}$, and $D_{w}^{\bar{k}} \cdot l^{1}(p, w) \neq D_{n}^{\bar{k}} \cdot l^{2}(p, w)$. By Taylor's theorem this is a contradiction to the fact that for all $n, v^{1}\left(p, w_{n}\right)=u\left(x_{n}^{1}\right)=u\left(x_{n}^{2}\right)=v^{2}\left(p, w_{n}\right)$. Q.E.D.

Remark. It is by no means necessary to consider derivatives only up to the order of $l+1$. Taking higher derivatives into account allows us to reduce the size of the set of exceptional utility functions consicierably.

\section{References}

Araujo. A. and A. Mas-6 well. 1978. Notes on the smoothing of aggregate demand. Journal (i) Mathematical Economics, 5, 1131?7.

Dierker. E., H. Dierker and $W$. Troce el, 1980. Smouthing demand by aggregation with re-pect to wealth. Journal of Mathematical Economics, forthcoming.

Golubitsky, M. and V. Guillemin 1973, Stable mappings and their singularities (Springer. Berlin).

Hildenbrand, W., 1974. Core and equilibria of a large econes..y (Princeton University Press. Princeton, NJ).

Mas.Colell, A. and W. Neuefeind, 1977, Some generic properties of aggregate excess demand and an application, Econometrica 45, 591599.

Sondermann, D. 1975, Smoothing demand by aggregation, Journal of Mathematic, Economics 201223.

Sc ndermann, D.. 1976. On a measure theorctical problem in mathematical econe nic. in: Springer Lecture Notes in Mathematics 541.

sondermann. D., 1980. Uniqueness of mean maximizers and continuity of aggregate emand. Journal of Mathematical Economics, fortheoming. 